\title{
Ärztliche Berufsbildung und Patientensicherheit
}

Was haben ärztliche Berufsbildung und Patientensicherheit gemeinsam? Ist dies ein Sauregurkenzeit-Thema?

Durch die ärztliche Berufsbildung sollen Ärztinnen und Ärzte die Kompetenz erhalten, Patientinnen und Patienten zu heilen, deren Leiden zu lindern oder sie in diesen zu begleiten. Primum nil nocere ist oberstes Primat.

Seit der Publikation von «To Err is Human: Building a Safer New Health System» [1] wird die Patientensicherheit durch Politik, Öffentlichkeit und Betroffene hinterfragt. Es wurde der alogische Begriff «Fehlerkultur» geschaffen, und dieser wurde nun durch «Sicherheitskultur» ersetzt. Dies ist im Einklang mit der Sicherheitsphilosophie von DuPont*.

Auf lokaler und nationaler Ebene wurden Stiftungen, multiple Aktivitäten und Instrumente geschaffen, um die Sicherheit ärztlichen Handelns per se und in Institutionen zu fördern. Es wurden verschiedene Instrumente entwickelt zur Erfassung von Komplikationen und Beinahefehlern sowie zu deren Auswertung im Hinblick auf eine Verbesserung der Patientenversorgung (u.a. CIRS, CIRRNET, Komplikationenliste SGIM). Schon früh wurde auf die Bedeutung der Pharmakotherapie als eines der wichtigsten Faktoren der Patientensicherheit aufmerksam gemacht. Die Kommission für Weiter- und Fortbildung (KWFB) der FMH forderte als Lernziel in allen klinischen Weiterbildungsprogrammen die Vermittlung der Kompetenz zur rationalen und sicheren Pharmakotherapie sowie des Wissens über die Arzneimittelprüfung und der Kenntnis der gesetzlichen Vorschriften über die Anwendung der Arzneimittel, namentlich im Bereich der obligatorischen Krankenpflegeversicherung. Diese inzwischen erfüllte Forderung wurde vom Departement des Innern aus ökonomischen Gründen hinterfragt, was aus Sicht der Patientensicherheit unverständlich ist.

Die Ärzteschaft forderte wiederholt elektronische Entscheidungshilfen zur Unterstützung der Pharmakotherapie und die elektronische Arzneimittelverschreibung. Die dazu notwendigen Investitionen erfolgten zögernd, da die Ressourcen prioritär in den buchhalterischen Bereichen der Institutionen eingesetzt wurden. Die Sicherheit der Patienten wird der Verwaltung und dem Rechnungswesen leider hintangestellt.

Aufgrund der Visitation von über 150 Weiterbildungsstätten muss festgehalten werden, dass die Sicherheitskultur in vielen Institutionen ein grosses Steigerungspotential aufweist. Die Arbeitsgruppe «Patientensicherheit» der Schweizerischen Akademie der Medizinischen Wissenschaften (SAMW) fordert einen Ausbau der Anstrengungen im Bereich der Patientensicherheit in der ärztlichen Berufsbildung. Die Sicherheit der Patienten soll dadurch auch bei der ärztlichen Berufsbildung an vorderste Stelle gerückt werden.

Wir benötigen dringend Investitionen in die Sicherheitskultur, damit wir Patientinnen und Patienten heute schon sicher behandeln können und auch in Zukunft über entsprechend geschulte Ärztegenerationen verfügen. Somit hängen ärztliche Berufsbildung und Patientensicherheit eng voneinander ab.

Dr. med. Max Giger, Mitglied des Zentralvorstandes der FMH, Präsident der Kommission für Weiterund Fortbildung der FMH

1 Kohn LT, Corrigan JM, Donaldson MS (editors). To Err is Human: Building a Safer New Health System. Washington, D.C.: National Academic Press; 2000.

* Im Anschluss an einen schweren Arbeitsunfall mit Schiesspulver entwickelte der Industriekonzern DuPont ein Sicherheitsmanagement zur Vermeidung von Arbeitsunfällen. DuPont wurde zu einem der sichersten Arbeitgeber und vermarktete später dieses Arbeitssicherheitsmanagement. Dessen Prinzipien können von der Medizin übernommen werden. 